\title{
Selective Effects of Cholinergic Modulation on Task Performance during Selective Attention
}

\author{
Maura L Furey*,', Pietro Pietrini ${ }^{2}$, James V Haxby ${ }^{3}$ and Wayne C Drevets' \\ 'Mood and Anxiety Disorders Program, National Institute of Mental Health, National Institutes of Health, Bethesda, MD, USA; ${ }^{2}$ Laboratory of \\ Clinical Biochemistry and Molecular Biology, University of Pisa, Pisa, Italy; ${ }^{3}$ Department of Psychology, Princeton University, Princeton, NJ, USA
}

\begin{abstract}
The cholinergic neurotransmitter system is critically linked to cognitive functions including attention. The current studies were designed to evaluate the effect of a cholinergic agonist and an antagonist on performance during a selective visual attention task where the inherent salience of attended/unattended stimuli was modulated. Two randomized, placebo-controlled, crossover studies were performed, one $(n=9)$ with the anticholinesterase physostigmine $(1.0 \mathrm{mg} / \mathrm{h})$, and the other $(n=30)$ with the anticholinergic scopolamine $(0.4 \mathrm{mc} / \mathrm{kg})$. During the task, two double-exposure pictures of faces and houses were presented side by side. Subjects were cued to attend to either the face or the house component of the stimuli, and were instructed to perform a matching task with the two exemplars from the attended category. The cue changed every 4-7 trials to instruct subjects to shift attention from one stimulus component to the other. During placebo in both studies, reaction time (RT) associated with the first trial following a cued shift in attention was longer than RT associated with later trials $(p<0.05)$; RT also was significantly longer when attending to houses than to faces $(p<0.05)$. Physostigmine decreased RT relative to placebo preferentially during trials greater than one $(p<0.05)$, with no change during trial one; and decreased RT preferentially during the attention to houses condition $(p<0.05)$ vs attention to faces. Scopolamine increased RT relative to placebo selectively during trials greater than one $(p<0.05)$, and preferentially increased RT during the attention to faces condition $(p<0.05)$. The results suggest that enhancement or impairment of cholinergic activity preferentially influences the maintenance of selective attention (ie trials greater than I). Moreover, effects of cholinergic manipulation depend on the selective attention condition (ie faces vs houses), which may suggest that cholinergic activity interacts with stimulus salience. The findings are discussed within the context of the role of acetylcholine both in stimulus processing and stimulus salience, and in establishing attention biases through top-down and bottom-up mechanisms of attention.

Neuropsychopharmacology (2008) 33, 913-923; doi: I0.1038/sj.npp. I30 I46I; published online 30 May 2007
\end{abstract}

Keywords: physostigmine; scopolamine; acetylcholine; human; cognition

\section{INTRODUCTION}

Selective attention constitutes the ability to discriminate significant or relevant stimuli from irrelevant stimuli (ie noise) and to process information within our environment preferentially (Desimone, 1998; Desimone and Duncan, 1995; Kastner and Pinsk, 2004; Moran and Desimone, 1985). The need for such a selective process results from a limited attentional capacity, so that the simultaneous presentation of multiple stimuli produces a competition for representation at the neural level (Desimone, 1998; Kastner et al, 1998). Single-unit recording studies (Chelazzi et al, 1998; Desimone, 1998; Reynolds et al, 1999) and functional brain imaging studies (Beck and Kastner, 2005; Deco and Rolls,

*Correspondence: Dr ML Furey, Mood and Anxiety Disorders Program, National Institute of Mental Health, National Institutes of Health, I5K North Drive, Room I I5B, Bethesda, MD 20892, USA, Tel: + I 301594 777, Fax: + I 301594 9959, E-mail: mfurey@mail.nih.gov Received 2 March 2007; revised 13 April 2007; accepted I6 April 2007
2005; Kastner et al, 1998) have demonstrated that the processing of a visual stimulus is influenced by the presence of other, unattended visual stimuli, whereby neighboring stimuli interact to produce suppressive effects on the processing of a target stimulus. The biased competition model of selective attention suggests that this neural competition among multiple stimuli can be mediated by two mechanisms that each contribute to the biasing of attention, including 'bottom-up' processes that act through stimulus-based operations, and 'top-down' processes that act through cognitive or executive actions (Desimone, 1998; Duncan, 1998). Bottom-up stimulus-based attentional mechanisms refer to neural processing that is biased by stimuli with inherently salient or meaningful features, so that attention is captured automatically and is associated with an enhancement of the neural representation of the stimulus. Inherent stimulus salience may result from the presence of stimulus characteristics that produce sensory salience (eg high contrast; unique color) (Egeth and Yantis, 1997; Parkhurst et al, 2002) or from the presence of stimuli 
that potentially have biological relevance (ie behaviorally or socially) or environmental significance (Friesen et al, 2004; Ohman, 2005; Parkhurst et al, 2002; Vuilleumier et al, 2001). In either case, attention is captured automatically and is associated with an enhancement of the neural representation of the stimulus. Top-down attentional mechanisms refer to knowledge-based processes where attention is oriented intentionally, resulting in the enhancement of neural representations of relevant, goal-directed stimuli and improved discrimination among competing stimuli based on biases toward targets (Connor et al, 2004; Sarter et al, 2001). Bottom-up and top-down mechanisms then interact (Corbetta and Shulman, 2002; Reynolds and Desimone, 2003; Sarter et al, 2001) to result in a biased neural representation of a stimulus.

Human faces are socially important stimuli that convey critical information regarding the environment. Evidence suggests that human faces capture attention or are given higher priority than other types of objects (Bindemann et al, 2005; Ro et al, 2001; Vuilleumier, 2000) and may be processed automatically (Bredart et al, 2006; Lavie et al, 2003; Ohman, 2002; Palermo and Rhodes, 2006). Researchers have demonstrated that human faces produce more interference when presented as distractor stimuli than do nonface objects (Bindemann et al, 2005; Ro et al, 2001), and some have suggested that faces are difficult to ignore due to their biological and social importance (Lavie et al, 2003). Together these findings suggest that faces may be preferentially processed, perhaps through a combination of bottom-up and top-down processes that bias neural processing in favor of faces.

The literature is rich with evidence of the involvement of the cholinergic system in attention mechanisms (Everitt and Robbins, 1997; Hasselmo and McGaughy, 2004; Himmelheber et al, 2000; Robbins, 1997; Sarter and Bruno, 2000; Sarter et al, 2003, 2005b; Yu and Dayan, 2002). Researchers have hypothesized that attentional processes are mediated through cholinergic mechanisms that facilitate the processing of sensory information (Robbins, 1997) and some evidence exists to support this idea (Furey et al, 2000b; Sillito and Kemp, 1983a). Furthermore, evidence indicates that the cholinergic system is recruited through both bottom-up, stimulus driven mechanisms and by top-down, goal-directed mechanisms suggesting that cholinergic involvement in stimulus processing reflects the combined influence of both bottom-up and top-down attentional processes (reviewed by Sarter et al, 2005a).

In general, the cholinergic neurons of the basal forebrain that projects throughout neocortex are thought to enhance signal-to-noise $(\mathrm{S} / \mathrm{N})$ ratios for neural processing (Murphy and Sillito, 1991; Sato et al, 1987). Sillito (Sillito and Kemp, 1983b) demonstrated that the direct application of acetylcholine to cat visual cortex increased the selectivity of the cell's response to stimulus orientation, consistent with the hypothesis that acetylcholine increases S/N. Similarly, Buzsaki (1989) showed that cholinergic input to hippocampus is inhibitory, suggesting that acetylcholine may enhance $\mathrm{S} / \mathrm{N}$ in hippocampus by reducing the response to noise. The cholinergic influence on $\mathrm{S} / \mathrm{N}$ may constitute the neural mechanism through which the cholinergic system influences selective attention, and may establish the relative strengths of the neural representations of competing stimuli. For example, a functional imaging study (Furey et al, 2000b) demonstrated that enhanced cholinergic activity selectively increased neural responses to taskrelevant stimuli (ie signal) with reduced or no change in neural responses to task-irrelevant stimuli (ie noise), consistent with a selective enhancement for target stimuli via $\mathrm{S} / \mathrm{N}$ processing. The modulation of $\mathrm{S} / \mathrm{N}$ processing in the context of the neural representation of competing stimuli may alter the stimulus bias established between attended and unattended stimuli.

The purpose of the current study was to evaluate the influence of cholinergic modulation on behavioral performance of a selective attention task. The task required the alternation of attention between two object categories present in each stimulus, thus the task required the shifting of goal-directed attention between two competing stimuli. The two object categories differed in the level of inherent salience (faces and houses), and thus as the task required the shift in directed attention, the salience of the unattended stimulus changed. We expected that acetylcholine is central to establishing the processing bias among competing stimuli. We were interested specifically in evaluating the influence of cholinergic modulation on goal-directed performance, and predicted that performance would change differentially depending on the inherent salience of the unattended stimulus so that changes in performance would reflect any shift in stimulus bias among the competing stimuli. A stimulus category bias would be reflected by the difference in reaction times (RTs) when attending to each of the two stimulus categories, and the larger this RT difference the greater the bias toward the stimulus category with the fastest RT. Two separate studies were conducted, one evaluating the enhancement of cholinergic activity using the anticholinesterase and physostigmine, and the other investigating the inhibition of cholinergic function using scopolamine.

\section{EXPERIMENT 1: EFFECT OF ENHANCING CHOLINERGIC ACTIVITY ON SELECTIVE ATTENTION}

\section{Methods}

Subjects. Nine medically and psychiatrically healthy individuals (mean age $\pm S D=31 \pm 6$ years; four females/five males) participated in a randomized, double-blind, placebocontrolled crossover study. All participants gave written informed consent after the purpose of the study and potential side effects of the drug were fully explained. The study was approved by the National Institute on Aging Institutional Review Board (NIH protocol 00-M-0056).

Experimental design. Subjects participated in two testing sessions, during which they received in random order i.v. infusions of placebo or drug and subsequently performed the selective attention task (see below). Participants received an infusion of physostigmine at a rate of $1.0 \mathrm{mg} /$ $\mathrm{h}$ according to the following procedure: a 10-min loading dose of $1.93 \mathrm{mg} / \mathrm{h}$ was used to quickly achieve the desired plasma levels followed by a maintenance drip of $0.816 \mathrm{mg} / \mathrm{h}$ to maintain stable drug levels to completion of the study session. Infusions continued for $30 \mathrm{~min}$ before beginning the task to obtain stable drug effects (Furey et al, 2000a). 
Glycopyrrolate, which is a cholinergic muscarinic antagonist that does not cross the blood-brain barrier, was administered i.v. $(0.02 \mathrm{mg})$ before the physostigmine infusion to minimize peripheral side effects (Mirakhur et al, 1977; Oduro, 1975). For the placebo infusions, the same infusion schedule was used with saline. Blood pressure and electrocardiogram (ECG) were monitored throughout each session for each subject.

Task design. Subjects performed a matching task while viewing two stimuli shown simultaneously, side by side. In the selective attention task condition, two double-exposure pictures of faces and houses were presented. Subjects were instructed by a cue to attend to either the face or the house component of the stimuli, and to decide if the two exemplars from the attended category were of the same person or house. Pictures with different views of the same person or house were used, so that subjects could not base their response on a simple pattern match. Subjects were cued to shift their attention from one stimulus component to the other every 4-7 trials (see Figure 1a). Stimuli were presented for $2.5 \mathrm{~s}$ and were followed by a $1.5 \mathrm{~s}$ intertrial interval. In the control task condition, two stimuli were presented in the same spatial and temporal manner but here the double-exposure images were phase scrambled to create stimuli that had equivalent luminance, contrast, and spatial frequency spectra as the double-exposure pictures. The control condition required subjects to determine if the scrambled images were the same or different. To control for the presentation of the cue in the attention task, a cue comprised of one large and one small ' $x$ ' (ie $X x$ ) was presented between the two scrambled image stimuli. The cue changed every 4-7 trials by switching the location of the large and small $\mathrm{x}(\mathrm{eg} \times \mathrm{X})$, with no relevance to task requirements. Performance RT and accuracy were recorded.

Data analysis. Before analysis, any RT that was less than $800 \mathrm{~ms}$ was considered an error and excluded from the data set, and any measure that exceeded three SDs above the mean was excluded as an outlier. RT and accuracy data were analyzed using repeated measures analysis of variance
(ANOVA) under placebo conditions to characterize baseline response features to the attentional target (faces $v s$ houses) and the trial order (responses to the first trial after a shift in attention to characterize performance associated with shifting selective attention; and responses to subsequent trials to characterize performance associated with the maintenance of selective attention, while subjects retain attention to one target category). RT and accuracy associated with the maintenance of selective attention were obtained by averaging response measures together across trials 1-4 before data analysis. Trials 5-7 were not included, as these trials did not occur following every attention shift. Drug effects were also analyzed by using repeated measures ANOVA so that drug condition (placebo $v s$ physostigmine), attention target, and trial were assessed. In the presence of significant interactions or main effects, $t$-test comparisons were used to further characterize these effects.

\section{Results}

Under placebo, RT showed a significant effect of attentional target $(\mathrm{F}=16.5, p=0.004)$, where responses were slower when individuals attended to houses than when they attended to faces. A significant effect of trial order also was observed $(\mathrm{F}=10.15, p=0.01)$, with slower responses to trial 1 than to trials greater than 1 . Accuracy data showed a significant attentional target $\times$ trial order interaction under placebo conditions $(\mathrm{F}=14.5, p=0.005)$, where accuracy was greater to the first trial $v s$ later trials when attending to faces, but accuracy was greater to later trials than to the first trial when attending to houses.

Under physostigmine, a significant drug effect was present $(\mathrm{F}=7.3, p=0.027)$ indicating an overall reduction in $\mathrm{RT}$ during drug $($ mean $\pm \mathrm{SD}=1755 \pm 259)$ relative to placebo $(1819 \pm 256.7)$. A significant $\mathrm{drug} \times$ trial order interaction also was observed $(\mathrm{F}=5.3, p=0.05)$ showing that RT was reduced during physostigmine to a greater extent for later trials than for trial 1 (Figure 2a), with $t$-tests demonstrating that no significant change in trial 1 occurred while a significant reduction was observed to trials greater than 1. A trend toward a significant drug $\times$ attention a Shifting Attention:
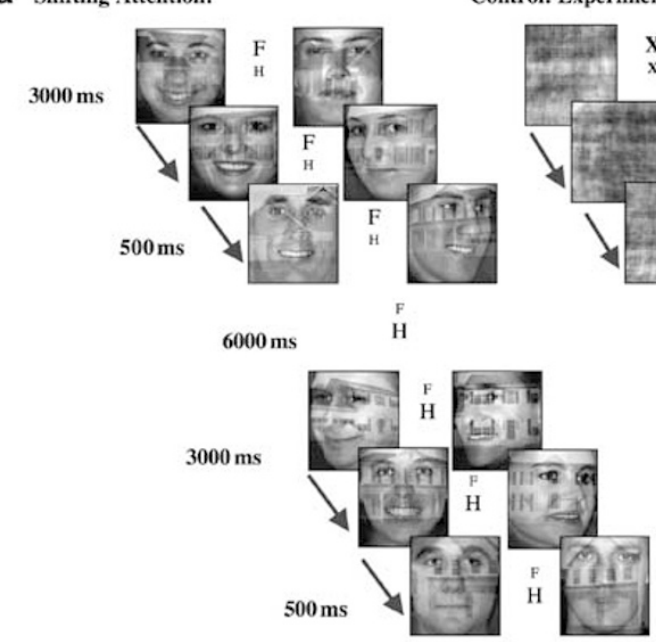

Control: Experiment 1

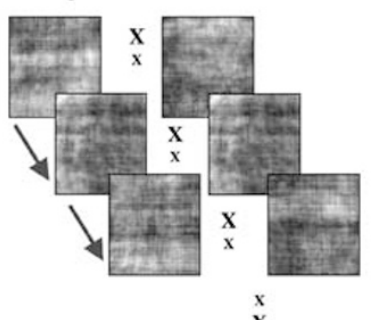

$\mathrm{X}$

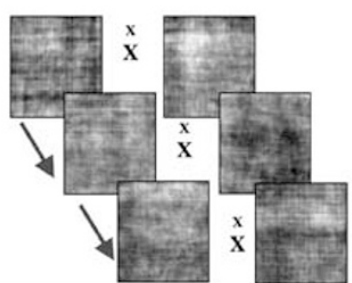

b Control: Experiment 2
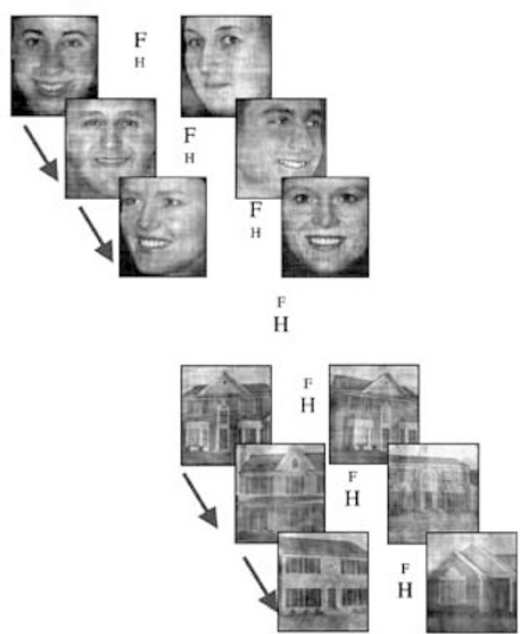

Figure I The selective attention task. 
a

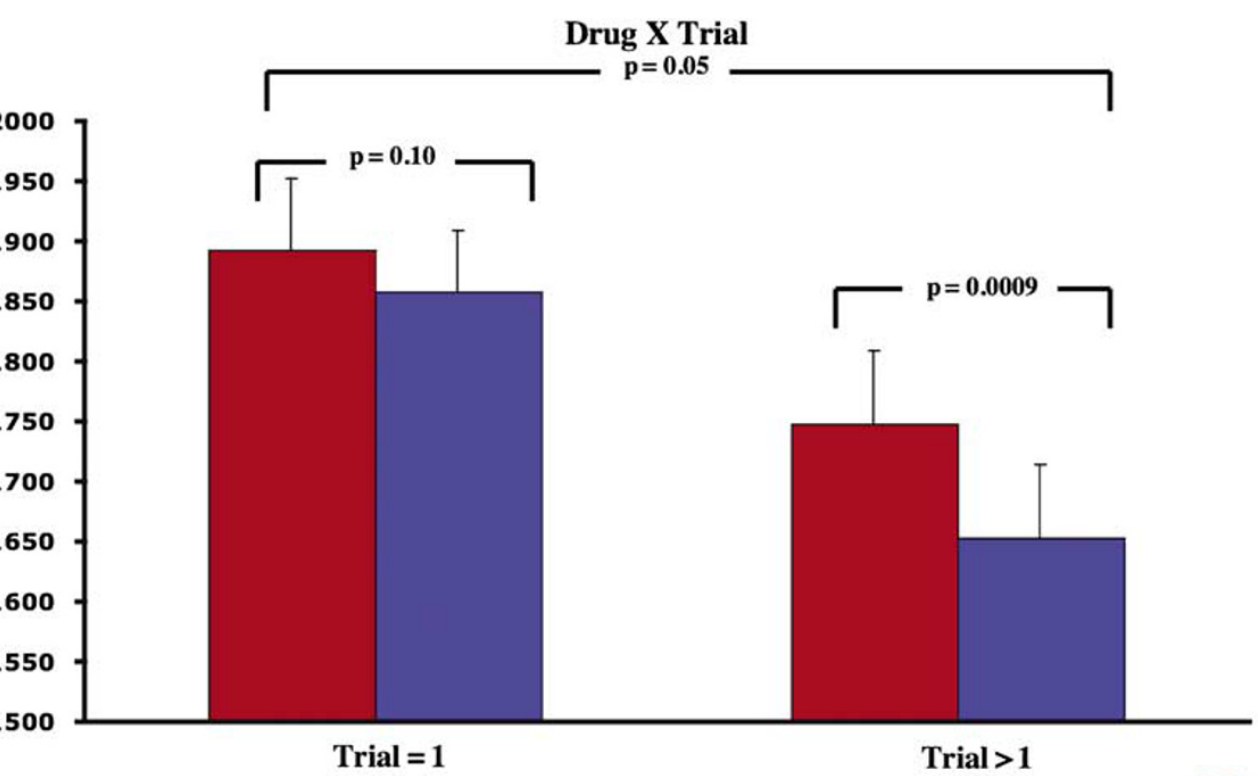

b

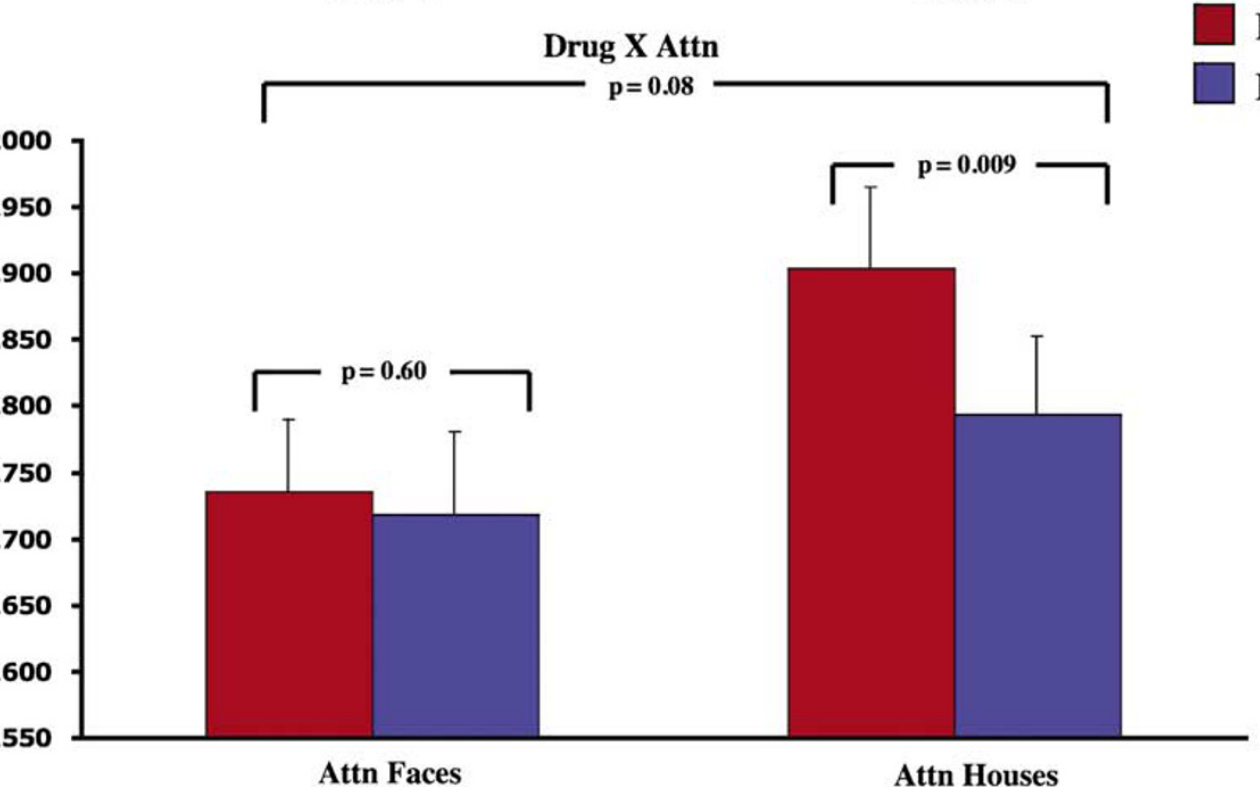

Trial >1

Figure 2 The effects of drug on mean RTs ( + SE) are shown for trial order (trial I vs subsequent trials) in (a) and for selective attention (faces vs houses) in (b). Performance during placebo is indicated in red and during physostigmine in blue. The p-values indicate level of significance for the identified interactions and for the within condition drug effects.

interaction was seen ( $\mathrm{F}=4.01, p=0.078$; Figure $2 \mathrm{~b})$ with reductions in $\mathrm{RT}$ when attending to houses $(p=0.009)$ but not when attending to faces $(p=0.60)$. Consistently, a significant drug condition $\times$ attention $\times$ trial order interaction also was observed $(\mathrm{F}=5.7, p=0.04)$ (Figure 3$)$, demonstrating that physostigmine decreased RT differentially based on the attention conditions, relative to trial type. Specifically, within the attention to faces condition, a significant drug $\times$ trial order interaction $(\mathrm{F}=8.64$, $p=0.002$ ) was present, showing that relative to placebo, physostigmine preferentially produced a reduction in RT to later trials, consistent with the overall drug $\times$ trial order interaction reported above. In contrast, within the attention to houses condition, a decrease in RT during drug relative to placebo was evident $(\mathrm{F}=21.54, p=0.002)$, but no interaction with trial was present $(\mathrm{F}=0.2, p=0.67)$, indicating that RT reductions occurred to all trials.
No overall drug effect on performance accuracy was observed $(\mathrm{F}=0.01, p=0.92)$. A trend toward a drug $\times$ attention $\times$ trial order interaction was present $(\mathrm{F}=4.0$, $p=0.08)$, suggesting that the attention $\times$ trial interaction seen under the placebo condition diminished under physostigmine. No change in accuracy was seen in the later trials for either attention condition.

No change $(p=0.30)$ in RT was observed to the control task during physostigmine (mean $\pm S D=1284 \pm 275)$ relative to placebo $(1267 \pm 242)$.

\section{Discussion}

Cholinergic enhancement with physostigmine improved selective attention, but preferentially enhanced performance during maintenance of selective attention as compared to shifting the target of selective attention. This enhancement 


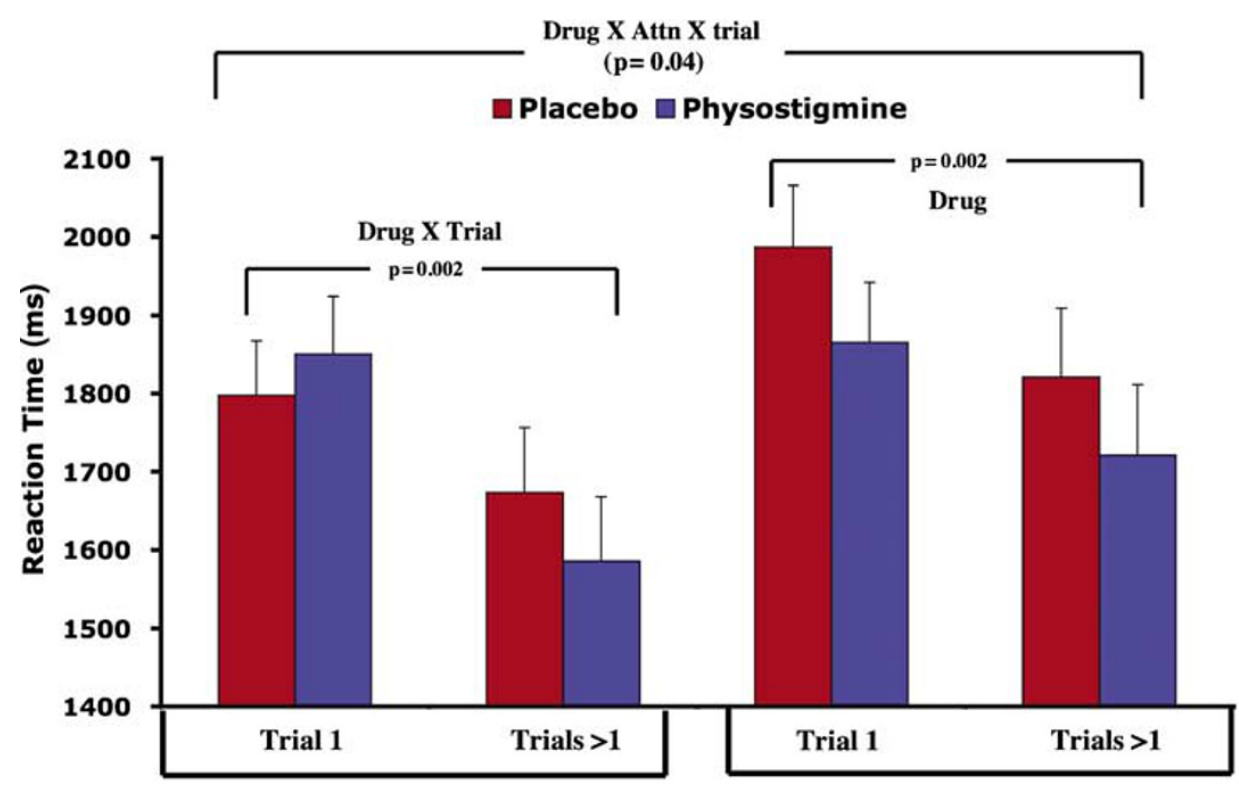

Attn Faces

Attn Houses

Figure 3 Mean RTs ( + SE) characterizing the drug $\times$ attention condition by trial order interaction, with performance during placebo in red and during physostigmine in blue.

differed based on the attention condition however, an effect that may be explained by cholinergic modulation of the relative salience of competing stimuli.

'Shift cost' is a term that defines the behavioral cost associated with shifting attention from one stimulus component to another, and is reflected by an increase in $\mathrm{RT}$ and decrease in performance accuracy in association with the first trial following a shift in selective attention relative to the subsequent trials (Monsell, 2003; Rogers and Monsell, 1995; Wylie and Allport, 2000). This observation suggests that the cognitive requirements may differ at different points in the task. Researchers have suggested that this response pattern develops as a result of an incomplete cognitive reconfiguration, so that performance observed during trial 1 occurs before a complete reconfiguration of the cognitive task set (Monsell, 2003; Rogers and Monsell, 1995). The results reported here show that cholinergic enhancement primarily influenced the maintenance of selective attention (ie trials greater than the first trial after shifting attention) with less of an influence on shifting selective attention per se (ie the first trial following a shift in selective attention). Our results may imply that the cholinergic effect observed during trials greater than 1 is specific to performance that follows the completion of cognitive reconfiguration, and is unrelated to the processes involved in cognitive reconfiguration. The process of changing or shifting the task set thus would be uninfluenced by cholinergic function.

Physostigmine also preferentially modulated performance during the attention to houses condition, when faces were unattended and presumably producing interference to the processing of houses (Bindemann et al, 2005; Lavie et al, 2003; Ro et al, 2001). From the perspective of the influence of unattended stimuli, faces are favored to grab attention and produce interference over some other forms of stimuli, with the result that when stimuli are competing for attention, a bias for faces exists (Bindemann et al, 2005).
In the attention to houses condition in the absence of cholinergic modulation, the face would produce interference at the expense of the house while in the attention to face condition, the house would not produce a similar level of interference. The current results suggest that the enhancement of cholinergic activity may modulate the relative salience of the face and house stimulus components so that the interference of the unattended face stimulus during attention to houses is altered, producing a shift in the face bias with the result being that the relative salience of the house stimulus is increased. Alternatively, the results may imply that enhanced cholinergic activity may influence top-down mechanisms by improving the ability to disengage attention or to ignore a salient stimulus that is not task related.

These results lead to clear hypotheses regarding the expected behavioral effects of inhibiting cholinergic activity. Specifically, we expected that blocking cholinergic activity preferentially would impair performance during the maintenance of selective attention as opposed to the shifting of selective attention. Moreover, we hypothesized that the processing bias toward faces observed at baseline would shift during scopolamine. During physostigmine we observed a shift in the face bias resulting principally from improvement in performance during attention to the unfavored house component of the stimulus. Following cholinergic inhibition, we predicted a shift in the face bias resulting from diminished selectivity for the favored face stimulus.

\section{EXPERIMENT 2: EFFECT OF INHIBITING CHOLINERGIC ACTIVITY ON SELECTIVE ATTENTION}

\section{Methods}

Subjects. Thirty healthy individuals (mean age $\pm \mathrm{SD}=30 \pm 7$ years; 17 females/13 males) participated in a randomized, 
double-blind crossover study. All participants gave written informed consent after the purpose of the study and potential side effects of the drug were fully explained. The study was approved by the National Institute of Mental Health Institutional Review Board.

Experimental design. Subjects participated in multiple testing sessions, during which they received in random order i.v. infusions of placebo or drug and subsequently performed the shifting selective attention task (see below). Participants received an infusion of scopolamine at a dose of $0.04 \mu \mathrm{g} / \mathrm{kg}$ over a $15 \mathrm{~min}$ infusion period (Ebert et al, 2001). Following completion of the infusion, a 45-min waiting period ensued to allow the peak cognitive effects to develop and to allow the peak side effects (ie drowsiness) to diminish (Safer and Allen, 1971). The same infusion and waiting periods were used with saline for the placebo infusions. Blood pressure and ECG were monitored throughout each session for each subject.

Task design. Subjects performed the matching task in the selective attention paradigm as described above. In the control task (Figure 1b), two stimuli were presented in the same spatial and temporal manner but images from each stimulus category were superimposed on phase-scrambled images of the other category to create stimuli that had equivalent luminance, contrast, and spatial frequency spectra as the double-exposure pictures. The control condition required subjects to determine if the intact pictures were of the same person or the same house. A cue was presented between the two stimuli that changed every 4-7 trials at which time the category of the intact component of the stimulus shifted. Performance RT and accuracy were recorded.

Data analysis. Statistical methods are as described for Experiment 1.

\section{Results}

Under placebo conditions, RT showed a significant effect of attention $(\mathrm{F}=87.3, p<0.0001)$ where $\mathrm{RT}$ was slower when individuals attended to houses than when they attended to faces, and a significant effect of trial $(\mathrm{F}=4.5, p=0.044)$ with slower RTs associated with the first trial following a shift in attention $v s$ the subsequent trials. Accuracy data showed a trend toward a trial effect $(\mathrm{F}=3.2, p=0.08)$ with accuracy tending to be higher to trials greater than 1 relative to the first trial following a shift. No other effect was significant under placebo conditions.

Under scopolamine, no overall drug effect on RT was present $(\mathrm{F}=0.84, \quad p=0.37)$. A significant $\operatorname{drug} \times$ trial interaction was observed $(\mathrm{F}=12.8, p=0.001)$ with scopolamine selectively increasing RT to trials greater than 1 , with no change in the first trial following a shift in attention (Figure 4a). As we hypothesized that scopolamine would modulate performance preferentially during the attention to faces condition, we also performed separate analyses on performance RT when attending to faces and houses, and found that RT increased preferentially when attending to faces $(F=6.5, p=0.02)$ with no significant effect when attending to houses $(\mathrm{F}=1.5, p=0.24)$ (Figure $4 \mathrm{~b})$, although the drug $\times$ attention interaction was not significant. There was no drug effect on performance accuracy, although there was a trend for scopolamine to decrease accuracy overall $(\mathrm{F}=2.8, p=0.10)$. Scopolamine effects on RT $(p>0.2)$ and accuracy $(p>0.2)$ did not differ based on gender.

Under placebo conditions in the control task, where stimulus conditions included intact faces and houses superimposed on phase-scrambled pictures, there was no significant difference in RT based upon trial (ie trial $1 v s$ trials greater than 1$)(\mathrm{F}=0.31, p=0.58)$ or based on the attentional target (ie faces or houses) $(\mathrm{F}=2.1$, $p=0.13$ ). A significant difference in performance accuracy was observed based on attentional target $(\mathrm{F}=11.7$, $p<0.002$ ), with accuracy to houses being higher than accuracy to faces.

There was no significant overall effect of scopolamine on $\mathrm{RT}(\mathrm{F}=0.30, p=0.59)$ in the control task. A trend toward a drug $\times$ stimulus condition interaction $(\mathrm{F}=3.0, p=0.095)$ was observed; in direct comparisons between placebo and scopolamine for each of the stimulus conditions, a significant decrease in RT for house stimuli was seen during drug $(\mathrm{F}=5.4, p=0.02)$, while no change during face stimuli was observed $(\mathrm{F}=0.01, p=0.94)$ (Figure $5 \mathrm{a})$. There was no significant overall effect of scopolamine on performance accuracy $(\mathrm{F}=1.6, p=0.29)$ during the control task. A drug $\times$ stimulus condition interaction was observed $(\mathrm{F}=6.9, p=0.01)$, and was explained by a selective reduction in accuracy during face trials, while there was no change during house trials $(\mathrm{F}=0.15, p=0.67)$ (Figure $5 \mathrm{~b}$ ). A drug $\times$ stimulus condition $\times$ trial interaction $(\mathrm{F}=7.8, p=0.009)$ was also seen, demonstrating that the selective reduction in accuracy observed during face trials $(\mathrm{F}=28.1, p=0.0001)$ was larger to trial 1 than to trials greater than 1.

Performance on the attention task was compared to that on the control task to characterize the specificity of the drug effects on selective attention. A significant drug $\times$ task interaction was detected $(\mathrm{F}=4.8, p=0.04)$, that showed increased RT specifically to the selective attention task, with no increase in the control task (Figure 5c). A significant drug $\times$ task $\times$ trial interaction $(F=6.0, p=0.02)$ also was evident demonstrating that the larger increase in RT during the selective attention task occurred preferentially during trials greater than 1. A trend toward a drug $\times$ task $\times$ stimulus condition interaction was present $(\mathrm{F}=3.4$, $p=0.07)$ due to the selective reduction in accuracy during trials with faces in the control task. No other difference in the drug effect on the task conditions was seen.

Post hoc analyses were also performed to compare directly the behavioral effects of physostigmine and scopolamine. Thus drug condition (placebo and drug), attention, trial, and drug group (physostigmine and scopolamine) were assessed in these analyses. A significant drug condition $\times$ trial $\times$ drug group interaction was found $(F=10.31$, $p=0.003$ ), and demonstrated that the drug effects were largest for later trials ( $v s$ trial 1), where scopolamine increased and physostigmine decreased RT. This result is summarized in Figure $6 \mathrm{a}$ where the difference between RT during placebo and drug is shown for trial 1 and for trials greater than 1, for both physostigmine and scopolamine. The differential effects of physostigmine and scopolamine 

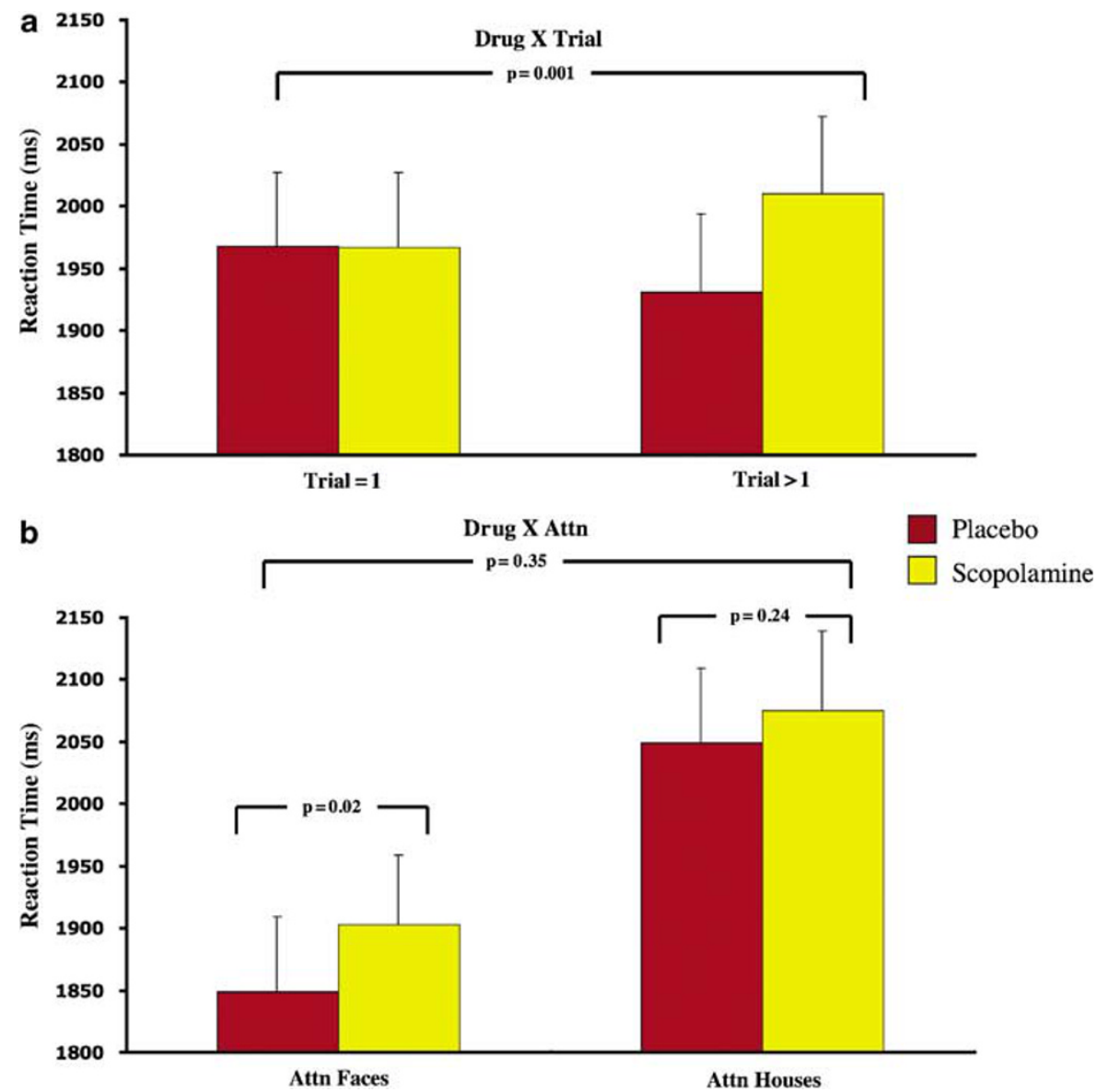

Figure 4 The effects of drug on mean RTs (+ SE) are shown for trial order (trial I vs subsequent trials) in (a) and for selective attention (faces vs houses) in (b). Performance during placebo is indicated in red and during scopolamine in yellow. The p-values indicate level of significance for the indicated interactions and for the within condition drug effects.

on the attention conditions are shown in Figure $6 \mathrm{~b}$ where the difference between RT during placebo and drug is shown during attention to faces and houses. Scopolamine increases RT, with significant increases seen only during attention to faces; physostigmine decreases RT, with significant reductions seen only during attention to houses. The drug condition $\times$ attention $\times$ drug group interaction was not significant, but a significant drug condition $\times$ attention $\times$ trial $\times$ drug group interaction was seen $(\mathrm{F}=4.0, p=0.05)$.

\section{Discussion}

The results from this study suggest that cholinergic modulation preferentially influences maintenance of selective attention, with little influence on the shifting of attention. Enhancing cholinergic function using physostigmine improved task performance primarily in trials beyond the first trial following a shift in attention, suggesting that the effects were predominantly associated with maintenance of selective attention. Parallel to the physostigmine result, inhibiting cholinergic function using scopolamine impaired performance primarily in trials associated with maintenance of selective attention. Together these findings provide complementary data showing that cholinergic function is related preferentially with the maintenance of selective attention, after task-set reconfiguration has taken place (Monsell, 2003), rather than during the shifting of attention per se.

Our findings also support the hypothesis that manipulation of cholinergic activity modulates the relative salience of competing stimuli during selective attention to result in specific and selective effects on performance. Further, our results support the hypothesis that the cholinergic system, which is instrumental in the perceptual processing of stimuli, contributes to establishing stimulus biases at a neural level based on the interaction between stimulusdriven and goal-directed mechanisms that modulate the representation of competing stimuli. Under both conditions of cholinergic modulation, the bias toward face processing observed under placebo was diminished during drug, as indicated by a reduction in the differences between the RT when attending to faces and when attending to houses. The face bias was diminished during physostigmine by improving performance primarily when attending to the less favored stimulus (ie houses), rendering the performance during the two attention conditions more similar. In contrast, the face processing bias was diminished during scopolamine by reducing performance primarily when attending to the favored stimulus (ie faces), again rendering performance during the two attention conditions more similar. 

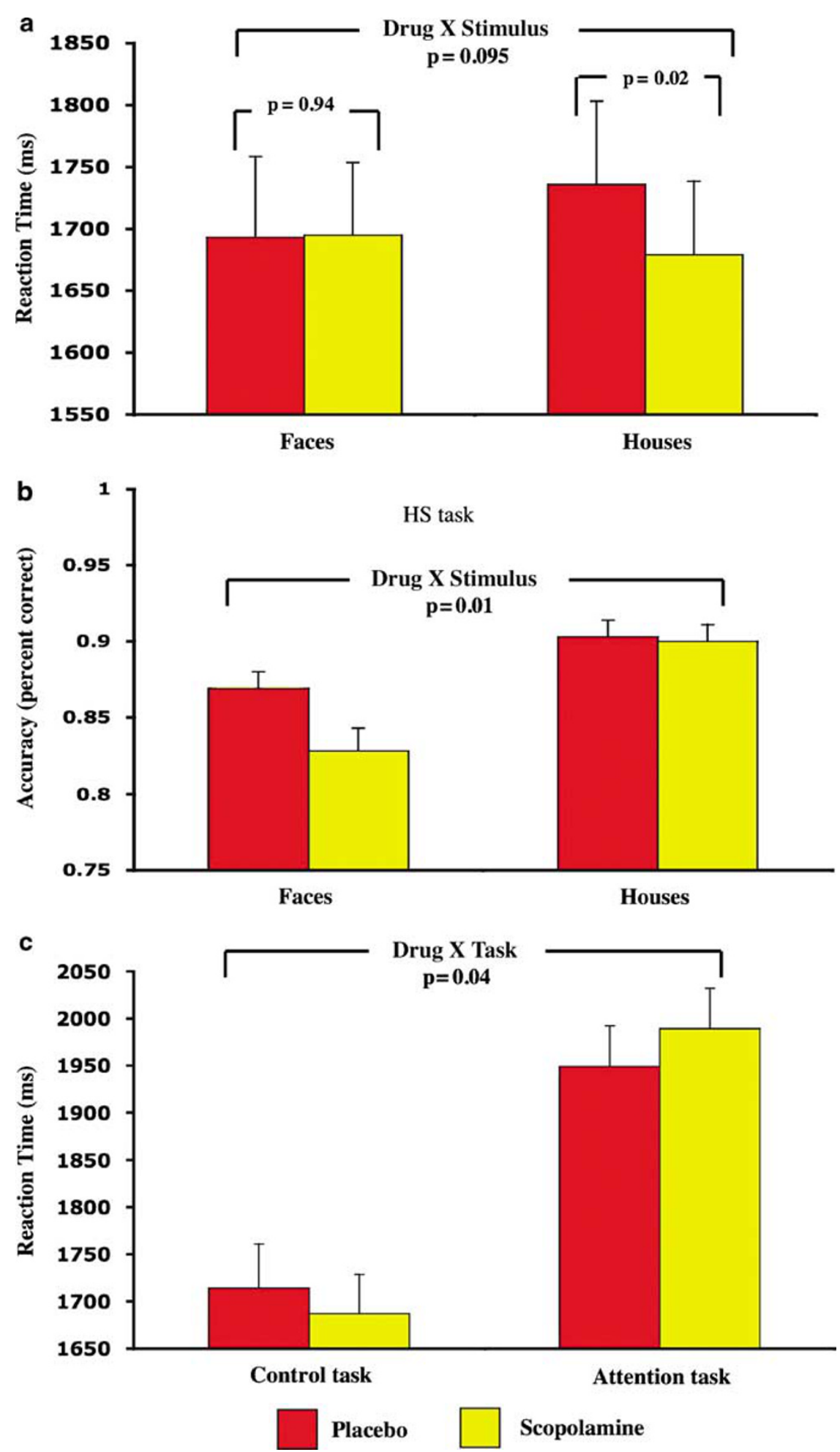

Figure 5 The effects of drug on mean RT ( \pm SE) (a) and performance accuracy (b) during the control task are shown, separated for trials with faces and trials with houses on phase-scrambled backgrounds. Performance during placebo is indicated in red and during scopolamine in yellow. The p-values indicate level of significance for the indicated interactions and for the within condition drug effects.

Previously we provided an alternative interpretation of the physostigmine results, suggesting that enhanced cholinergic activity may act exclusively through top-down attention control mechanisms, and improve the ability to disengage attention or to ignore a salient stimulus that is not task related. The scopolamine results would argue against this interpretation. If enhancing cholinergic activity improved the ability to ignore a salient stimulus (ie faces) through improved top-down effects, then we would expect that impairing cholinergic activity would diminish top-down 

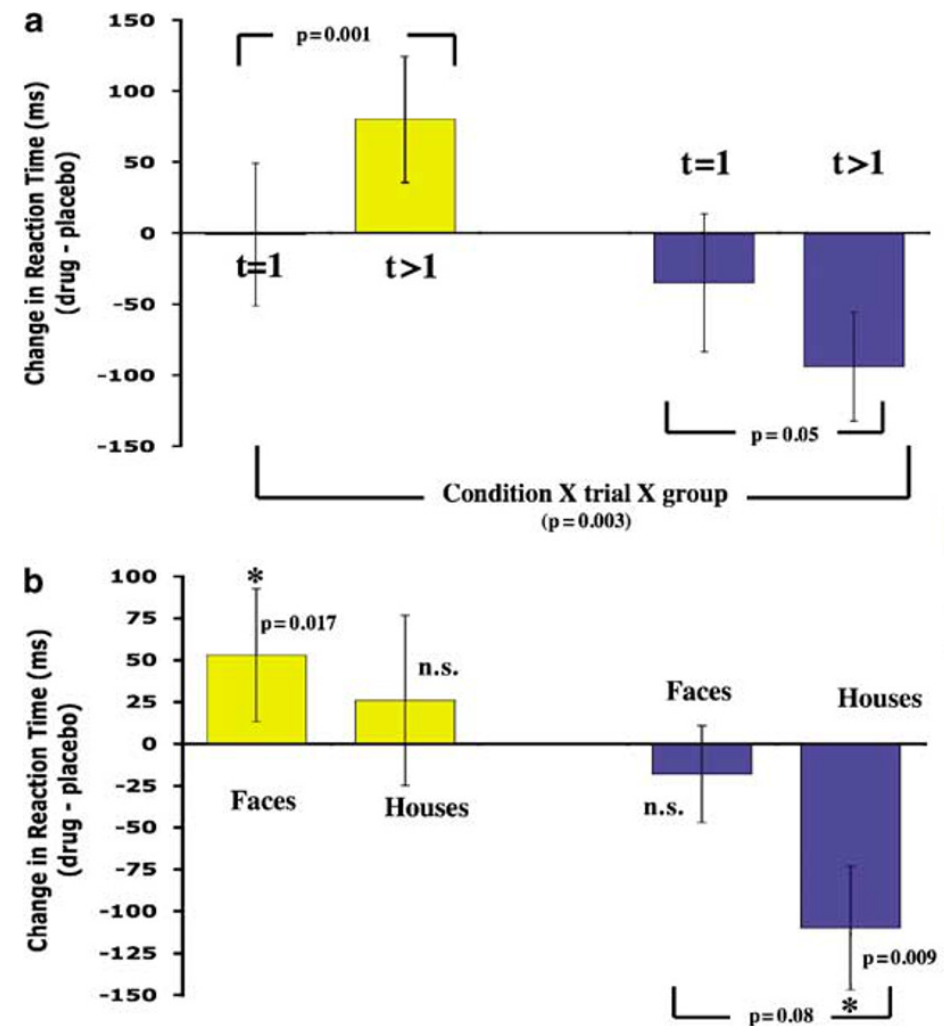

Scopolamine

Physostigmine

Figure 6 The mean changes in RTs ( + SE) on drug as compared to placebo are shown for scopolamine in yellow and for physostigmine in blue. The differential effects of drug (scopolamine vs physostigmine) are shown for trial order (a) and for selective attention (b). As drug effects primarily are found in trails subsequent to trial I, only these trials are included when assessing the effects of drug on selective attention (b).

abilities. This may present as a reduced ability to disengage attention, thus producing a generalized impairment in performance, or this may present as a reduced ability to ignore salient stimuli resulting in an increase in the processing bias toward faces, with RT primarily increasing during attention to houses. Instead, we see a selective reduction in the face bias, with a decrease in the face preference indicated by preferential increases in RT when attending to faces. The results together support the interpretation that acetylcholine influences stimulus processing through interactions between stimulus-driven and goal-directed mechanisms to produce a relative salience among the competing stimuli.

In the current studies under placebo conditions, a bias toward processing faces over houses was evident, as RT was faster during attention to faces than attention to houses. Conceivably this bias could be due to the manner in which the stimuli were developed, inadvertently resulting in the faces being easier to discriminate. However, the selectivity of the physostigmine effect, showing a preference for enhanced processing of the attended stimulus during attention to houses, suggests that the original bias is face specific and not due to an unintentional bias that resulted from the manner in which the stimuli were created. Similarly, the influence of acetylcholine on the face bias under placebo conditions also is diminished during scopolamine, as evidenced by changes in performance preferentially during the attention to faces condition. Again, the selectivity of the effect to the attention to faces condition argues against the interpretation that the behavioral response pattern is due to faces being easier to discriminate before drug administration. One would expect scopolamine to produce similar impairment in both attention conditions if there was no stimulus selectivity associated with these effects. Moreover, an attentional bias toward faces has been described by others, as demonstrated by the difficulty associated with the disengagement of processing resources from face stimuli (Bindemann et al, 2005) or the advantage that faces retain over other types of objects to capture attention (Ro et al, 2001; Vuilleumier, 2000). Finally, in the event that the original, baseline category bias was influenced by the manner in which the stimuli were developed, this would have little influence on the interpretation of the data. Specifically, the behavioral effects discussed in this paper are in the context of the direction and manner of response change from baseline following cholinergic modulation, and this behavioral change is interpreted in the context of stimulus bias.

Importantly, no overall effect of scopolamine on RT was observed. The effects we obtained were selective to task conditions, reflecting effects on stimulus processing speed, and were not attributable to nonspecific impairments in task performance.

The control task also provided critical information regarding the specificity of our findings. Again, no overall effect of scopolamine on RT or on accuracy was observed during performance of the control task. A selective reduction in performance accuracy was observed to faces, with no change in accuracy to trials with houses. In addition, we identified a decrease in RT during scopolamine 
specifically to the house stimuli, a result that further highlights both the selectivity of our findings to the selective attention condition as well as the absence of a generalized effect on performance. A similar specificity was observed during physostigmine, where RT reductions were selective to task conditions, with no effect observed during the control condition.

The basal forebrain cholinergic neurons release acetylcholine in response to target stimuli under conditions of directed attention and to inherently salient stimuli that automatically draw attention (Sarter et al, 2005a). The effect of cholinergic modulation on the processing of each competing stimulus during a selective attention task would depend on the relative influence of acetylcholine on these two interacting components. Evidence suggests that acetylcholine does influence both mechanisms (Sarter et al, 2005a), but the relative influence is not clear. Our findings are consistent with the interpretation that acetylcholine alters the relative salience of the signal (ie attended stimulus) and noise (ie unattended stimulus).

Together, these findings support the hypothesis that acetylcholine influences both stimulus-driven and taskdirected attentional mechanisms in an interactive way. Moreover, this framework merges well with the biased competition model of attention (Desimone, 1998; Reynolds et al, 1999), supporting the hypothesis that cholinergic activity influences stimulus-driven and task-directed attentional processing to establish biases among competing stimuli.

The effort to determine specifically which cholinergic receptor(s) mediates these behavioral effects is limited as a result of the mechanism of action of physostigmine. As an anticholinesterase inhibitor, physostigmine acts by inhibiting the breakdown of endogenously released acetylcholine present in the synapse. As a result, we are unable to determine directly whether physostigmine is acting through muscarinic or nicotinic receptor sites to produce the observed results. Because scopolamine interacts selectively with muscarinic receptors, we can attribute the behavioral effects on attention observed during scopolamine to muscarinic receptor antagonism. Moreover, that the behavioral effects of physostigmine and scopolamine observed in the context of this study are highly complementary, one might hypothesize that during this attention task, physostigmine is influencing cholinergic activity primarily at muscarinic receptor sites. Despite the fact that physostigmine is nonselective for receptor type, the nature of an anticholinesterase inhibitor is to enhance naturally released acetylcholine, so that the effects of the acetylcholine released while performing the selective attention task are bolstered. One could argue that we know muscarinic receptors are involved in performing this task based on the scopolamine results, and therefore conclude that physostigmine at least partly acts through muscarinic sites but may implicate nicotinic sites as well.

Interactions between estrogen and the cholinergic system (Granholm, 2000; Tinkler et al, 2004) are known to modulate cognition functions including attention (Granholm et al, 2002; Tinkler and Voytko, 2005; Voytko, 2002). In our post hoc analyses, we observed no difference in the behavioral response to scopolamine based on gender.

The results as reported here in the context of selective attention may be more specific to selective attention when face stimuli are involved. Evidence indicates that faces retain a unique ability to gain attention (Bindemann et al, 2005; Lavie et al, 2003; Ro et al, 2001), and in this regard the effects we have reported here may be specific to face stimuli.

These results suggest that acetylcholine contributes at multiple levels to the mechanisms underlying selective attention. The preferential influence on performance during later trials following a shift in attention during both physostigmine and scopolamine indicates that acetylcholine is more central to the maintenance of selective attention and less implicated in the shift in attention per se. The results also suggest that acetylcholine may influence selective attention via $\mathrm{S} / \mathrm{N}$ mechanisms that contribute to establishing relative salience among competing stimuli.

\section{ACKNOWLEDGEMENTS}

We thank Harvey Iwamoto for preparation of the selective attention task; Joanna Szczepanik, Jane Lange, Alice Liu, and Ashish Khanna for providing technical support, and the 5SW Day Hospital staff for nursing support.

\section{FINANCIAL DISCLOSURE/CONFLICT OF INTEREST}

Dr Furey and Dr Drevets have submitted a patent application for the use of scopolamine as an antidepressant and antianxiety agent in mood and anxiety disorders. The patent currently is pending. No other potential conflict of interest exists for any of the authors.

\section{REFERENCES}

Beck DM, Kastner S (2005). Stimulus context modulates competition in human extrastriate cortex. Nat Neurosci 8: 1110-1116.

Bindemann M, Burton AM, Hooge IT, Jenkins R, de Haan EH (2005). Faces retain attention. Psychon Bull Rev 12: 1048-1053.

Bredart S, Delchambre M, Laureys S (2006). Short article one's own face is hard to ignore. Q J Exp Psychol (Colchester) 59: 46-52.

Buzsaki G (1989). Two-stage model of memory trace formation: a role for 'noisy' brain states. Neuroscience 31: 551-570.

Chelazzi L, Duncan J, Miller EK, Desimone R (1998). Responses of neurons in inferior temporal cortex during memory-guided visual search. J Neurophysiol 80: 2918-2940.

Connor CE, Egeth HE, Yantis S (2004). Visual attention: bottom-up versus top-down. Curr Biol 14: R850-R852.

Corbetta M, Shulman GL (2002). Control of goal-directed and stimulus-driven attention in the brain. Nat Rev Neurosci 3: 201-215.

Deco G, Rolls ET (2005). Neurodynamics of biased competition and cooperation for attention: a model with spiking neurons. J Neurophysiol 94: 295-313.

Desimone R (1998). Visual attention mediated by biased competition in extrastriate visual cortex. Philos Trans R Soc Lond B Biol Sci 353: 1245-1255.

Desimone R, Duncan J (1995). Neural mechanisms of selective visual attention. Annu Rev Neurosci 18: 193-222.

Duncan J (1998). Converging levels of analysis in the cognitive neuroscience of visual attention. Philos Trans $R$ Soc Lond B Biol Sci 353: 1307-1317.

Ebert U, Grossmann M, Oertel R, Gramatte T, Kirch W (2001). Pharmacokinetic-pharmacodynamic modeling of the electroencephalogram effects of scopolamine in healthy volunteers. J Clin Pharmacol 41: 51-60. 
Egeth HE, Yantis S (1997). Visual attention: control, representation, and time course. Annu Rev Psychol 48: 269-297.

Everitt BJ, Robbins TW (1997). Central cholinergic systems and cognition. Annu Rev Psychol 48: 649-684.

Friesen CK, Ristic J, Kingstone A (2004). Attentional effects of counterpredictive gaze and arrow cues. J Exp Psychol Hum Percept Perform 30: 319-329.

Furey ML, Pietrini P, Alexander GE, Mentis MJ, Szczepanik J, Shetty U et al (2000a). Time course of pharmacodynamic and pharmacokinetic effects of physostigmine assessed by functional brain imaging in humans. Pharmacol Biochem Behav 66: 475-481.

Furey ML, Pietrini P, Haxby JV (2000b). Cholinergic enhancement and increased selectivity of perceptual processing during working memory. Science 290: 2315-2319.

Granholm AC (2000). Oestrogen and nerve growth factor-neuroprotection and repair in Alzheimer's disease. Expert Opin Investig Drugs 9: 685-694.

Granholm AC, Ford KA, Hyde LA, Bimonte HA, Hunter CL, Nelson $M$ et al (2002). Estrogen restores cognition and cholinergic phenotype in an animal model of Down syndrome. Physiol Behav 77: 371-385.

Hasselmo ME, McGaughy J (2004). High acetylcholine levels set circuit dynamics for attention and encoding and low acetylcholine levels set dynamics for consolidation. Prog Brain Res 145: 207-231.

Himmelheber AM, Sarter M, Bruno JP (2000). Increases in cortical acetylcholine release during sustained attention performance in rats. Brain Res Cogn Brain Res 9: 313-325.

Kastner S, De Weerd P, Desimone R, Ungerleider LG (1998). Mechanisms of directed attention in the human extrastriate cortex as revealed by functional MRI. Science 282: 108-111.

Kastner S, Pinsk MA (2004). Visual attention as a multilevel selection process. Cogn Affect Behav Neurosci 4: 483-500.

Lavie N, Ro T, Russell C (2003). The role of perceptual load in processing distractor faces. Psychol Sci 14: 510-515.

Mirakhur RK, Dundee JW, Clarke RS (1977). Glycopyrrolateneostigmine mixture for antagonism of neuromuscular block: comparison with atropine-neostigmine mixture. $\mathrm{Br}$ J Anaesth 49: 825-829.

Monsell S (2003). Task switching. Trends Cogn Sci 7: 134-140.

Moran J, Desimone R (1985). Selective attention gates visual processing in the extrastriate cortex. Science 229: 782-784.

Murphy PC, Sillito AM (1991). Cholinergic enhancement of direction selectivity in the visual cortex of the cat. Neuroscience 40: $13-20$.

Oduro KA (1975). Glycopyrrolate methobromide: 2. Comparison with atropine sulphate in anaesthesia. Can Anaesth Soc J 22: 466-473.

Ohman A (2002). Automaticity and the amygdala: nonconscious responses to emotional faces. Curr Direct Psychol Sci 11: 62-66.

Ohman A (2005). The role of the amygdala in human fear: automatic detection of threat. Psychoneuroendocrinology 30: 953-958.

Palermo R, Rhodes G (2006). Are you always on my mind? A review of how face perception and attention interact. Neuropsychologia 45: 75-92.

Parkhurst D, Law K, Niebur E (2002). Modeling the role of salience in the allocation of overt visual attention. Vision Res 42: 107-123.
Reynolds JH, Chelazzi L, Desimone R (1999). Competitive mechanisms subserve attention in macaque areas V2 and V4. J Neurosci 19: 1736-1753.

Reynolds JH, Desimone R (2003). Interacting roles of attention and visual salience in V4. Neuron 37: 853-863.

Ro T, Russell C, Lavie N (2001). Changing faces: a detection advantage in the flicker paradigm. Psychol Sci 12: 94-99.

Robbins TW (1997). Arousal systems and attentional processes. Biol Psychol 45: 57-71.

Rogers RD, Monsell S (1995). The cost of a predictable switch between simple cognitive tasks. J Exp Psychol Gen 124: 207-231.

Safer DJ, Allen RP (1971). The central effects of scopolamine in man. Biol Psychiatry 3: 347-355.

Sarter M, Bruno JP (2000). Cortical cholinergic inputs mediating arousal, attentional processing and dreaming: differential afferent regulation of the basal forebrain by telencephalic and brainstem afferents. Neuroscience 95: 933-952.

Sarter M, Bruno JP, Givens B (2003). Attentional functions of cortical cholinergic inputs: what does it mean for learning and memory? Neurobiol Learn Mem 80: 245-256.

Sarter M, Givens B, Bruno JP (2001). The cognitive neuroscience of sustained attention: where top-down meets bottom-up. Brain Res Brain Res Rev 35: 146-160.

Sarter M, Hasselmo ME, Bruno JP, Givens B (2005a). Unraveling the attentional functions of cortical cholinergic inputs: interactions between signal-driven and cognitive modulation of signal detection. Brain Res Brain Res Rev 48: 98-111.

Sarter M, Nelson CL, Bruno JP (2005b). Cortical cholinergic transmission and cortical information processing in schizophrenia. Schizophr Bull 31: 117-138.

Sato H, Hata Y, Masui H, Tsumoto T (1987). A functional role of cholinergic innervation to neurons in the cat visual cortex. J Neurophysiol 58: 765-780.

Sillito AM, Kemp JA (1983a). Cholinergic modulation of the functional organization of the cat visual cortex. Brain Res 289: 143-155.

Sillito AM, Kemp JA (1983b). Cholinergic modulation of the functional organization of the cat visual cortex. Brain Res 289: 143-155.

Tinkler GP, Tobin JR, Voytko ML (2004). Effects of two years of estrogen loss or replacement on nucleus basalis cholinergic neurons and cholinergic fibers to the dorsolateral prefrontal and inferior parietal cortex of monkeys. J Comp Neurol 469: 507-521.

Tinkler GP, Voytko ML (2005). Estrogen modulates cognitive and cholinergic processes in surgically menopausal monkeys. Prog Neuropsychopharmacol Biol Psychiatry 29: 423-431.

Voytko ML (2002). Estrogen and the cholinergic system modulate visuospatial attention in monkeys (Macaca fascicularis). Behav Neurosci 116: 187-197.

Vuilleumier P (2000). Faces call for attention: evidence from patients with visual extinction. Neuropsychologia 38: 693-700.

Vuilleumier P, Armony JL, Driver J, Dolan RJ (2001). Effects of attention and emotion on face processing in the human brain: an event-related fMRI study. Neuron 30: 829-841.

Wylie G, Allport A (2000). Task switching and the measurement of 'switch costs'. Psychol Res 63: 212-233.

Yu AJ, Dayan P (2002). Acetylcholine in cortical inference. Neural Netw 15: 719-730. 